\title{
Automated multi-well device to measure transepithelial electrical resistances under physiological conditions
}

\author{
Joachim Wegener, Dimitri Abrams, Wolfgang Willenbrink, Hans-Joachim \\ Galla, and Andreas Janshoff \\ Westfälische Wilhelms-Universität Münster, Münster, Germany
}

BioTechniques 37:590-597 (October 2004)

\begin{abstract}
Measurement of transendothelial or transepithelial electrical resistances (TERs) is a straightforward in situ experimental approach to monitor the expression or modulation of barrier-forming cell-to-cell contacts (tight junctions) in cultured cells grown on porous filters. Although widely accepted, there is currently no device available to automatically measure the time course of TERs under ordinary cell culture conditions $\left(37^{\circ} \mathrm{C}, 5 \%\right.$ or $\left.10 \% \mathrm{CO}_{2}\right)$. This paper describes a development from our laboratory that is capable of following in parallel the TERs of several filter-grown cell layers with time and in an entirely computer-controlled fashion. The cell cultures can be followed even in long-term experiments without any manual assistance or opening of the incubator. Besides reading TER values, this approach also returns the electrical capacitance of the cell layers, which is indicative of the expression of microvilli and other membrane extrusions. The device is based on reading the frequencydependent impedance of the cell layer, followed by equivalent circuit modeling to extract the cell-related parameters. It is compatible with several multi-well formats (up to 96 wells) and controlled by custom-designed software that reads, analyzes, and presents the data.
\end{abstract}

\section{INTRODUCTION}

The measurement of transepithelial electrical resistances (TERs) is a widely accepted and easy-to-perform experimental technique to determine the tightness of epithelial and endothelial cell layers in vitro (1-3). In multicellular organisms, these tissues serve as an active interface between two fluid compartments that limit free diffusion of solutes along the intercellular shunts and actively transport others along the transcellular route. Because TER values correlate favorably with the ability of barrier-forming cell layers to act as a diffusion barrier, they are often recorded to monitor and compare the establishment and modulation of barrier-forming cell-to-cell contacts (tight junctions; References 4-6).

To perform the measurement, the cells are cultured as monolayers on permeable filter supports that are commercially available with different pore densities and pore diameters $(0.4-8 \mu \mathrm{m})$, dependent on the manufacturer. This setup mimics the physiological location of endothelial and epithelial cell layers as interfacial tissue between two fluid compartments. When the characteristics of the filter support are properly selected, the cell layer is the diffusionlimiting entity, and experimental readings of the permeation rates for a given compound mirror the barrier properties of the cell layer. In order to measure the electrical resistance of such a filtergrown cell layer, electrodes are placed in the upper and lower medium compartments. Measuring the voltage drop across the cell layer upon injection of a defined electrical current allows the area-specific resistance of the cell layer to be deduced via Ohm's law, provided that the resistance of the empty filter insert has been determined independently and is subtracted (3). The beauty of the TER approach is its noninvasiveness and that measurements can be performed in less than a minute so that the status of the cell layer can be routinely monitored without any need to sacrifice the culture. However, there are also a few drawbacks: $(i)$ when the position of the electrodes or their geometry is not properly selected-as may apply when so-called chopstick electrodes (7) are used-the cells are exposed to an inhomogeneous electric field, often result- ing in an overestimation of TER (7); (ii) many researchers assume that TER readings exclusively reflect the status of the tight junctions. In case of very tight epithelia and endothelia, a considerable fraction of the total current will also pass the cell layer on transcellular pathways so that TER readings mirror the total resistance of transcellular and paracellular current pathways. Under these conditions, changes in TER cannot be assigned unequivocally to alterations in barrier-forming cell-to-cell contacts without further independent experiments (6). (iii) When very leaky cell layers, such as macrovascular endothelial cells, are examined, the measured TER may be dominated by inherent resistance contributions arising from the cell-substrate adhesion zone, as has been recently described (8). This contribution is negligible for cells with moderate or high paracellular tightness.

In spite of these limitations, which are important to recognize, TER measurements have been and will be an indispensable tool to learn more about epithelial and endothelial cell biology and to study the functional expression of barrier-forming cell-to-cell contacts (tight junctions) as well as their regulation. From the viewpoint of equipment, there is room for technical improvement. To the best of our knowledge, no apparatus has yet been described that provides the technical basis to perform time-resolved TER measurements for a set of cell-covered filter inserts under ordinary cell culture conditions in a humidified incubator and in an automated and entirely computer-controlled fashion. Here we describe a development from our laboratory that is designed to fill this gap. The device is custom-made to monitor the time course of TER even in long-term experiments and in various multi-well formats.

\section{MATERIALS AND METHODS}

\section{Apparatus Hardware Components}

The device described here consists of a custom-made multi-well measuring chamber (e.g., 12 or 24 well) that can be loaded with a corresponding number of cell-covered filter inserts. The elec- 
trodes, which are necessary to inject current and record voltage, are made from stainless steel and are integrated into the measurement chamber, as described in greater detail in the Results and Discussion section. The whole experiment is controlled by a commercial PC that is interfaced with a data acquisition board used for the electrical measurements (data acquisition DAQPad-6070E; National Instruments, Munich, Germany) and a set of relays that are used to address the individual filter inserts. The data acquisition board DAQPad-6070E is an external multifunctional device with two analog voltage output channels (D/A) and eight analog voltage-sensing channels (A/D) from which only two are used for the device described here. To control DAQPad-6070E and the relays, we wrote custom software in LabView ${ }^{\mathrm{TM}} 6$ (National Instruments). This software stores the raw data, controls the experiment timing, performs data analysis, and archives the data. Communication between the board and the $\mathrm{PC}$ is provided by a fire-wire interface (IEEE 1394). The software is available from the corresponding author upon request.

\section{Cell Culture}

Demonstration experiments were performed with primary cultured endothelial cells derived from porcine brain microvessels and primary cultured epithelial cells from the porcine choroid plexus. To run the measurements, both cell types were seeded on Costar $^{\circledR}$ Transwell $^{\circledR}$ filter inserts (Corning, Corning, NY, USA) with $10^{8}$ pores $/ \mathrm{cm}^{2}$ of $400 \mathrm{~nm}$ diameter each. After the capillary endothelial cells had reached confluence on the collagencoated filter inserts, the serum-containing medium was exchanged against serum-free Dulbecco's modified Eagle's medium (DMEM)/Ham's F12 medium [supplemented with $1.7 \mathrm{mM}$ glutamine and $1 \%(\mathrm{v} / \mathrm{v})$ penicillin/streptomycin] to induce final differentiation. The choroid plexus epithelial cells were seeded on laminin (from EHS mouse sarcoma) coated filter substrates (Sigma-Aldrich, Dreisenhofen, Germany). Confluent monolayers were also switched to serum-free DMEM/Ham's F12 medium [supplemented with $2 \mathrm{mM}$ glutamine and antibiotics as above] for differentiation. Additional cell culture details for both cell types have been previously described $(9,10)$.

\section{RESULTS AND DISCUSSION}

The core component of the new device is a measuring chamber that can be manufactured in several multi-well formats to be loaded with a corresponding number of cell-covered filter inserts. Figure 1A shows a photograph of a 12-well chamber. The bottom part $\mathbf{1}$ is made from simple plastics and serves as a frame to hold and fix the bottom stainless steel plate electrode $\mathbf{2}$, which is a common electrode for all wells. The individual wells are formed by a block of polytetrafluoroethylene (PTFE) 3 with corresponding holes. Figure 1B shows how a typical filter insert $\left(1.1 \mathrm{~cm}^{2}\right)$ fits into these wells. As shown in Figure 1A, the top part of the chamber 4 holds 12 individual stamplike electrodes $\mathbf{5}$ and fits exactly onto the lower part of the chamber so as to dip the individual stamp electrodes into the medium above the cell layer. The upper (dipping) electrodes are also made from stainless steel. Accordingly, in each well, the cell layer is sandwiched between the two electrodes as shown in Figure 1C and is exposed to a homogeneous electric field along the entire surface of the cell layer so that transepithelial impedance readings can be recorded accurately. The dipping electrodes are individually addressable by the software that controls the experiment so that we can measure all wells or any subset of the matrix in a sequential fashion. Figure $2 \mathrm{~A}$ shows a block diagram of the entire setup. The PC 1 controls the entire experiment and is connected $\mathbf{2}$ to the controller unit $\mathbf{3}$ housing the data acquisition board DAQPad-6070E and the relays. From the controller unit, contact $\mathbf{4}$ is made to the measurement chamber $\mathbf{5}$ inside the humidified incubator (Revco ${ }^{\circledR}$ Ultima $^{\mathrm{TM}}$; Kendro Laboratory Products, Asheville, NC, USA) by means of a ribbon cable.

In contrast to other approaches to measure TERs of cultured cells, the device presented here sequentially reads the electrical AC impedance of each filter insert at a preset number of frequencies in the range between 1 and $10^{5} \mathrm{~Hz}$. TER values are then subsequently extracted from these impedance spectra by equivalent circuit modeling.

The individual impedance measurement at a particular frequency is performed as follows. According to Ohm's law, the electrical impedance $\underline{Z}$ of the system (electrodes + cell covered filter insert + medium) can be calculated from the voltage drop $\underline{U}$ across the system and the associated current $\underline{I}$ running through it, thus $\underline{Z}=\underline{U} / \underline{I}$. To keep the measurement technically simple and to avoid current measurements, we have used the voltage divider shown in Figure 2B to determine the system impedance $Z$. The output of DAQPad$6070 \mathrm{E}$ applies a sinusoidal voltage of typically $45 \mathrm{mV}$ amplitude across the system and an external ohmic resistor $R$ arranged in series. Two voltage-sensing channels of DAQPad-6070E are used to read the amplitude of $(i)$ the voltage $\underline{U}_{\underline{O}}$ across the system and the extra resistance $R$ in series and (ii) the voltage

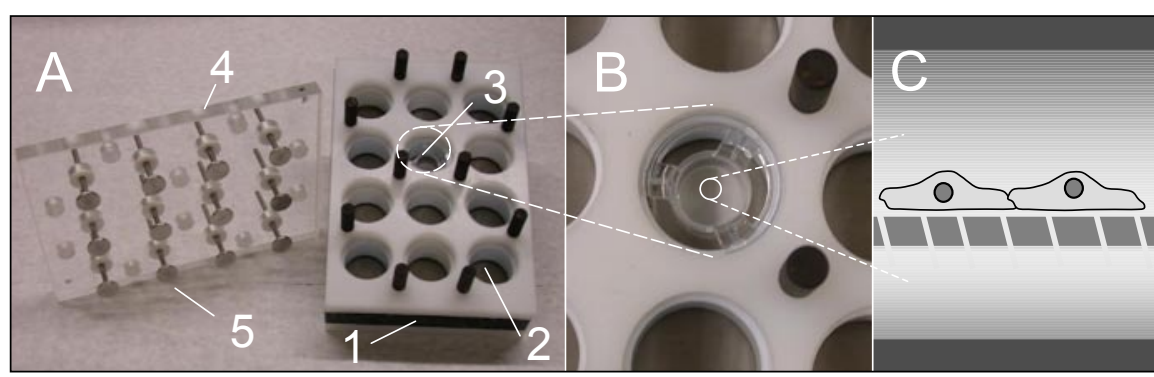

Figure 1. Design of the multi-well measurement chamber. (A) Photograph of the measurement chamber consisting of a common stainless steel electrode $\mathbf{2}$ integrated into the chamber bottom 1, a 12-well block that can be loaded with cell-covered filter inserts $\mathbf{3}$, and a lid $\mathbf{4}$ with 12 individually addressable dipping electrodes 5. (B) Detail of a filter insert $\left(1.1 \mathrm{~cm}^{2}\right.$ surface area) placed in one well of the chamber. (C) Diagram of the cell layer anchored to the porous filter membrane and sandwiched between the upper and lower electrodes. 
$\underline{U}_{s y s}$ across the system itself (compare Figure 2B). Both voltages ( $U_{s y s}$ and $U_{0}$ ) were measured together with their individual phase shifts relative to the total current $\left(\phi_{s y s}\right.$ and $\left.\phi_{0}\right)$. From these phasesensitive readings of the two voltages, we can calculate the complex impedance of the system $\underline{Z}_{\underline{s y \underline{s}}}$ according to:

$$
\boldsymbol{Z}_{s y s}=\boldsymbol{R} \cdot\left(\frac{1}{1-\frac{\boldsymbol{U}_{s y s}}{\boldsymbol{U}_{0}} \cdot \exp \left(\varphi_{s y s}-\varphi_{0}\right)}-1\right)
$$

[Eq. 1]

Only the magnitude of the system impedance $|Z|$ as a function of frequency is used for subsequent data analysis. In a typical experiment, we have measured the impedance of cell-covered filter inserts from $1 \mathrm{~Hz}$ to $100 \mathrm{kHz}$, reading 10 data points per frequency decade, where the points are equally spaced on a logarithmic scale (Figure $3 \mathrm{~A}$ ). The amplitude of the applied voltage was set to $45 \mathrm{mV}$ (root-meansquare). Thus, all technical requirements to perform impedance analysis in the frequency range needed here are provided by DAQPad-6070E plus an additional resistor $R$. It is noteworthy that the best value of $R$ depends on the total impedance of the cell-covered filter insert and, thus, mainly on filter size. In experiments presented here using filters of $1.1 \mathrm{~cm}^{2}$ surface area, $R$ was set to $200 \Omega$. The switch 6 on the front panel of the device housing 3 (Figure 2A) allows the value of $R$ to be changed if required by other experimental circumstances.

TER values of a given cell layer are then extracted from the impedance spectra by means of equivalent circuit modeling. This procedure holds several advantages from the technical viewpoint but also in terms of the information content of each experiment. Figure 3A shows the frequency-dependent magnitude of impedance $|Z|$ as typically recorded for endothelial or epithelial monolayers by this setup. In order to quantify the electrical parameters of the cell layer, we fit the transfer function of the equivalent circuit shown in Figure $3 \mathrm{~B}$ to the recorded data. The model network mirrors the electrical properties of the entire setup, including electrodes $\left(\mathrm{CPE}_{1,2}\right)$, medium $\left(R_{\text {med1,2 }}\right)$, and the cell layer (TER, $\left.C_{c l}\right)$, and allows quantification of their individual contributions. Here CPE stands for constant phase element, and a CPE is an empirical impedance element commonly used to describe the interface impedance between noble metal electrodes and electrolyte solutions $(11,12)$. The impedance of a CPE is given by $\underline{Z}_{C P E}=1 /\left[\mathrm{A} \cdot(\mathrm{i} \cdot \omega)^{\mathrm{n}}\right]$ with $\mathrm{n}$ between 0 and $1 . R_{\text {med }}$ represents the resistivity of the bulk electrolyte. With respect to the cell layer, it should be emphasized that the equivalent circuit modeling not only returns TER values as a measure of the cell layer's barrier function but also allows quantification of the cell layer's capacitance $\left(C_{c l}\right)$, which is indicative of the expression of membrane extrusions like microvilli and other membrane foldings. The solid line in Figure 3A shows the excellent agreement between measurement

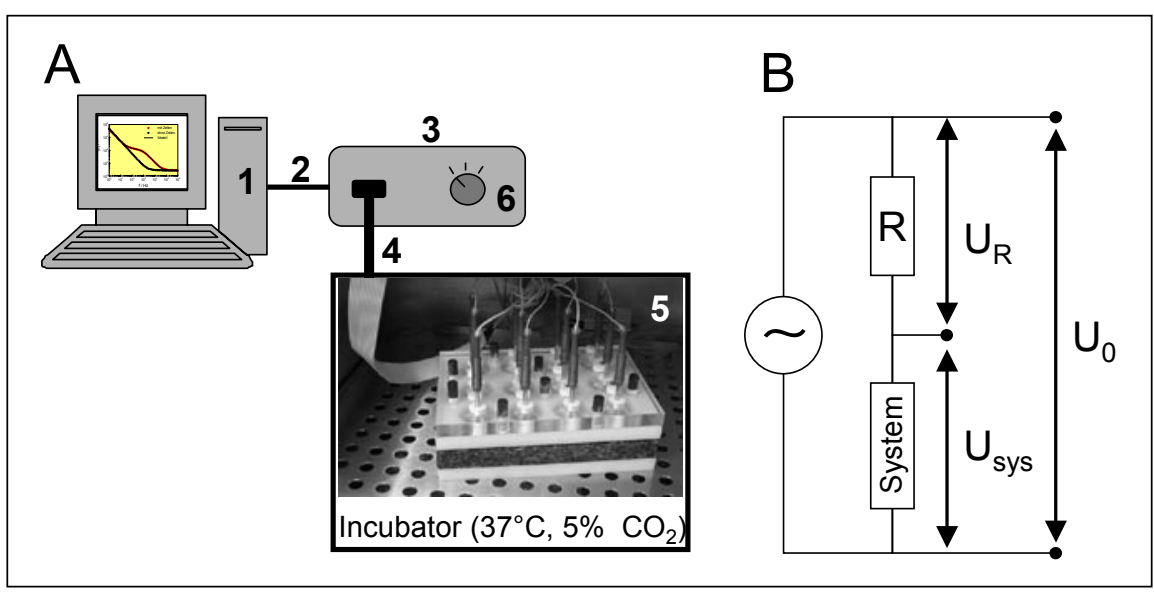

Figure 2. Experimental setup and circuit diagram. (A) Block diagram of the entire measurement setup with a PC 1, the data acquisition and relay unit $\mathbf{3}$, and the measurement chamber placed inside a humidified cell culture incubator 5 , including the appropriate electrical connections 2 and $\mathbf{4}$. Switch 6 allows for the adjustment of the series load resistor $R$, which is part of the electronic sensing circuit (see Figure 2B). (B) The voltage divider circuit used to read the system impedance. The oscillator of the data acquisition board applies a sinusoidal voltage of preset frequencies to the series arrangement of the electrochemical system (cells + electrode + medium) and the extra resistor $R$. From phase-sensitive readings of the voltages $U_{0}$ and $U_{s y s}$, we can calculate the impedance of the system.

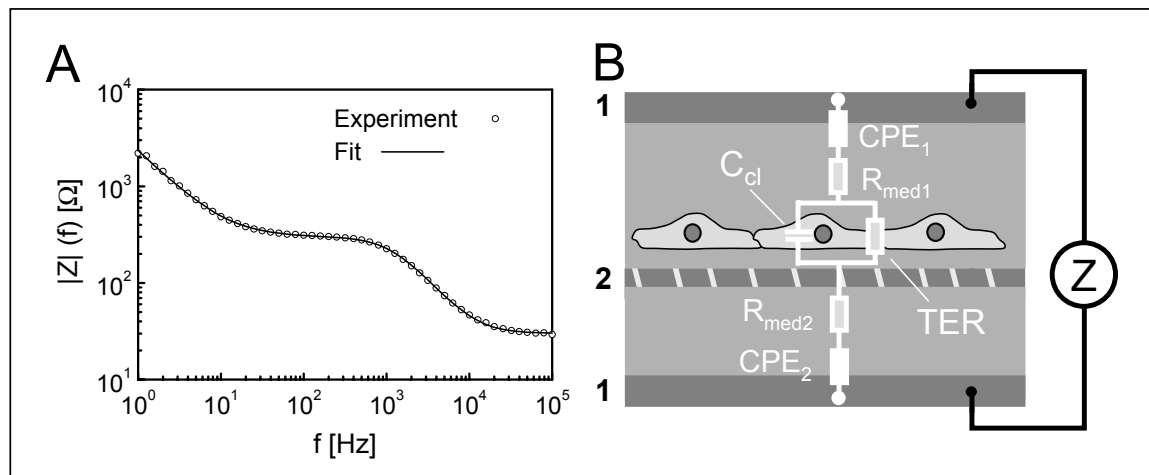

Figure 3. Equivalent circuit modeling of the raw data. (A) Frequency-dependent impedance magnitude of a cell-covered filter insert as recorded with the device presented here. IZI denotes the impedance magnitude, $f$, the frequency. The open circles represent experimental data whereas the solid line corresponds to the transfer function of the equivalent circuit shown in panel B after least-square optimization. The fit parameters are for this example: $R_{\text {med }}=24.0 \Omega$; transepithelial electrical resistance (TER) $=$ $382.7 \Omega \mathrm{cm}^{2} ; C_{c l}=0.44 \mu \mathrm{F} / \mathrm{cm}^{2} ;$ A (CPE) $=3.15 \cdot 10^{-5} \mu \mathrm{F} \cdot \mathrm{s}^{\mathrm{n}-1} / \mathrm{cm}^{2} ; n(C P E)=0.903$. (B) Diagram illustrating the electrical equivalent circuit that is used to extract TERs from frequency-dependent impedance readings. The constant phase elements $\mathrm{CPE}_{1}$ and $\mathrm{CPE}_{2}$ represent the impedance of the electrode/electrolyte interface. $R_{\text {med } 1}$ and $R_{\text {med } 2}$ stand for the medium resistance. TER and $C_{c l}$ characterize the electrical properties of the cell layer. CPE, constant phase element. 
and model. Fitting the parameters of the equivalent circuit to the experimental data is achieved via nonlinear leastsquare optimization according to the Levenberg-Marquardt algorithm (13). This fitting routine is an integral part of the software and is carried out immediately after the impedance spectrum of a particular filter has been completed. Thus, in a typical experiment, the software directly returns the time course of TER and $C_{c l}$, even for long-term experiments, without the need to open the incubator door. Mathematically, the equivalent circuit elements $R_{\text {med } 1}$ and $R_{\text {med } 2}$ as well as $\mathrm{CPE}_{1}$ and $\mathrm{CPE}_{2}$ cannot be distinguished from each other and are thus represented by one global impedance element $\left(R_{\text {med }}\right.$ or CPE, respectively) within the transfer function. Accordingly, the parameter $R_{\text {med }}$ represents the total medium resistance (including filter pores), whereas the parameters of the CPE mirror the electrical properties of the upper and lower electrode combined. This equivalent circuit and its rationale have already been introduced and discussed recently in detail (14). The hardware and the data analysis routine were further validated by measuring electrical networks (data not shown) made from well-defined electrical components whose impedance characteristics simulate those of cell-covered filter inserts.

The advantages of these data recording and data analysis procedures are as follows. (i) The measurement returns two parameters to describe the properties of the cell layer, TER and $C_{c l}$. Whereas the TER quantifies the cell layer's barrier function, the cell layer capacitance reflects predominantly the membrane surface area. Because it has been consistently found for many different cell types that an unfolded plasma membrane has a specific capacitance of roughly $1 \mu \mathrm{F} / \mathrm{cm}^{2}$, those values measured for differentiated and unperturbed cells reflect how much the membrane is folded, such as in epithelial microvilli (15). Knowledge of the true membrane surface area is important, for example, when active transepithelial transport processes are studied. The measured transport rates are directly affected by the available exchange area. (ii) The spectrometric TER approach also avoids the necessity to measure empty filter inserts because this is included in the measurement and independently quantified as $R_{\text {med }}$. When the medium conductivity changes during the course of an experiment (e.g., due to the addition of a drug or temperature gradients), this is monitored in the time course of $R_{\text {med }}$. (iii) In contrast to other techniques, such as the widespread chopstick electrode arrangement, we do not have to use one electrode pair to inject the current and another pair to determine the corresponding voltage drop across the cell layer. Instead, we use just one pair of electrodes for the entire measurement. This approach is justified as long as there is no significant faradaic current across the electrode/electrolyte interface. For the stainless steel electrodes used here, this is valid for voltage amplitudes up to $500 \mathrm{mV}$ (data not shown), which is more than 10 -fold the voltage applied in cell experiments. However, as a direct consequence of using a two-electrode setup, the electrical properties of the electrodes contribute to the system and have to be taken into account (CPE) when the raw data are analyzed to extract TER values. This extra effort, however, provides an indicator for electrode stability along the entire experiment. The parameters of the CPE that are quantified for each well in each impedance spectrum are followed over time at no extra cost and can be reviewed at any stage.

To demonstrate the capabilities of the new device, we present experimental data recorded for primary cultured endothelial cells derived from porcine brain microvessels and epithelial cells isolated from porcine choroid plexus. These two cell types are used extensively as cell culture models of the blood-brain (9) and the blood-cerebrospinal fluid barrier $(10,16)$. Figure 4A shows the time course of TER values for confluent porcine brain capillary endothelial cells when they were incubated in serum-free medium (SFM) 

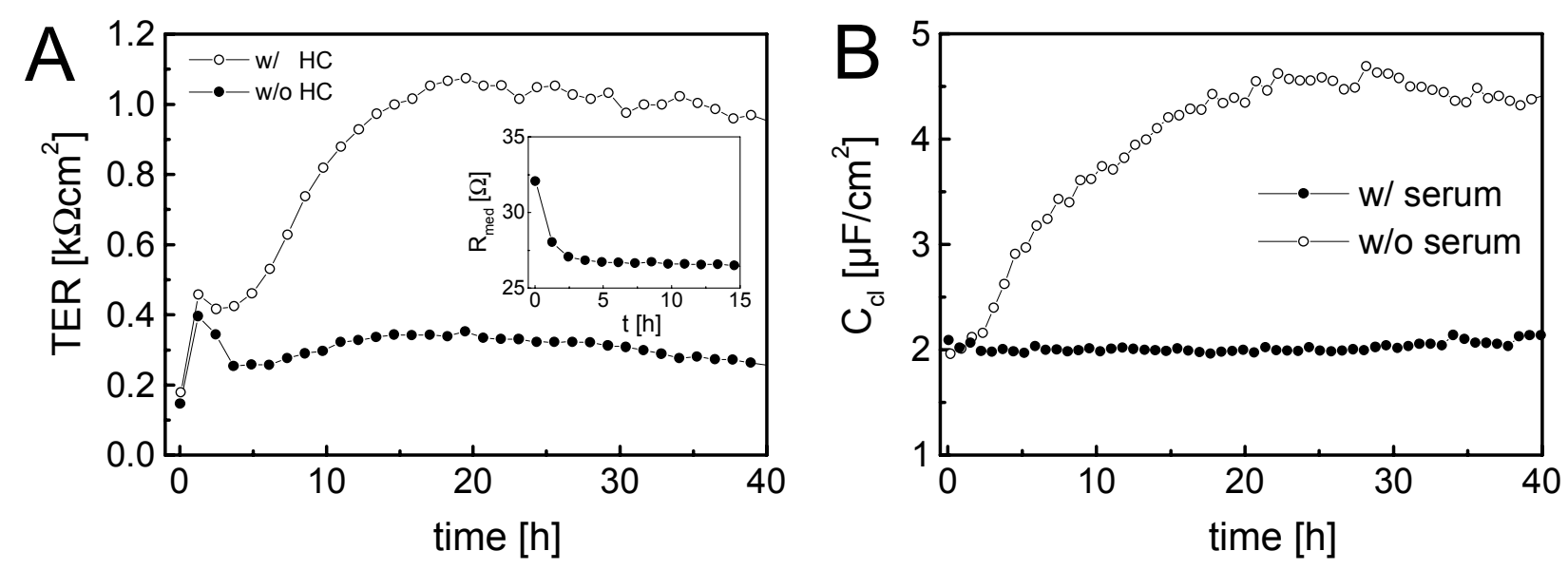

Figure 4. Time-resolved monitoring of transepithelial electrical resistance (TER) and membrane capacitance. Time course of TER for primary cultured endothelial cells derived from porcine brain microvessels when they are incubated in serum-free medium supplemented with $550 \mathrm{nM}$ hydrocortisone (open circles) or without hydrocortisone (filled circles). The insert shows the time course of the medium resistance $R_{m e d}$ throughout the first $15 \mathrm{~h}$ of the experiment. (B) Time course of the cell layer capacitance $C_{c l}$ for primary cultured epithelial cells derived from porcine choroid plexus when confluent cell layers are incubated in serum-free medium (open circles) or in serum-containing medium (filled circles).

with or without $550 \mathrm{nM}$ hydrocortisone. It has been previously reported that cerebral endothelial cells respond to hydrocortisone with a pronounced barrier strengthening (17). As expected, Figure $4 \mathrm{~A}$ shows that the TER increases with time for cell layers incubated in the presence of hydrocortisone (open circles) compared to the respective controls without hydrocortisone (filled circles). With the new device, we could follow the kinetics of this differentiation process in a totally undisturbed cell culture system for more than $45 \mathrm{~h}$ with a time resolution that was set to $30 \mathrm{~min}$ in this experiment. If needed, this time resolution can be significantly increased. With the settings specified above (1 to $10^{5} \mathrm{~Hz}, 10$ data points per decade), the system requires roughly $1 \mathrm{~min}$ measuring time per filter insert, giving a time resolution of $12 \mathrm{~min}$ in a 12-well setup. By reducing the number of filters measured in one run or the number of data points per spectrum, the performance may be improved further. The inset in Figure 4A shows the time course of the medium resistance $R_{\text {med }}$ for the first $15 \mathrm{~h}$ of the experiment. Because the medium resistance is a good indicator of the temperature of the cell culture medium inside the chamber, the initial jumps in TER values can be attributed to warming up of the medium (the chamber had been loaded with cells in a flow hood and was then placed into the incubator). Consistent with earlier electron microscopic studies, no significant changes were observed in the cell layer capacitance (data not shown) with or without hydrocortisone. The average membrane capacitance (twice the capacitance of the entire cell layer $C_{c l}$ since there are two membranes in series) of capillary endothelial cells amounts to $0.9-1 \mu \mathrm{F} / \mathrm{cm}^{2}$ and confirms an almost entirely unfolded membrane. Similar capacitance readings have been made for endothelial cells derived from different organs and/or species $(3,18,19)$.

Figure 4B shows the capacitance time course for two layers of choroid plexus epithelial cells that were incubated either with serum-containing medium or SFM after they had reached confluence. It is a striking feature of choroid plexus epithelial cells that they develop longer and more densely packed microvilli on their apical surface when incubated in SFM. This has been independently confirmed by scanning electron microscopy (10). Only under serum-free conditions do they express the phenotype of an actively transporting epithelium. Time course readings of the membrane capacitance allow this differentiation process to be followed noninvasively in situ. The membrane capacitance has become a valuable indicator in our laboratory to compare the differentiation of these cells in vitro from one preparation to the next.

The device presented here is compatible with various multi-well formats up to 96 wells. Each format certainly requires a new measuring chamber, but the concept of their design is the same as described above. By reducing the number of data points per spectrum or the frequency range that is used for analysis, the time resolution can be improved to allow meaningful kinetic experiments even in 96-well format. The device can be used to automatically monitor the time course of TER, even in experiments that run for several days, and may serve as a valuable tool in all studies addressing epithelial or endothelial cell biology or pharmacology.

\section{ACKNOWLEDGMENTS}

We would like to thank Andrej Dirks for his assistance in maintaining and updating the software as well as Sabine Huewel, Patrick Zeni, and Susanne Angelow for their expert help with the primary cultures. We also would like to acknowledge Daniela Rehder and Ines Nasdala (Max-Planck Institute for Vascular Biology Münster) for intensive testing and many helpful suggestions.

\section{COMPETING INTERESTS STATEMENT}

The authors declare no competing interests. 


\section{REFERENCES}

1.Diamond, J.M. 1977. The epithelial junction: bridge, gate and fence. Physiologist 20:10-18.

2.Cereijido, M., L. Gonzalez-Mariscal, R.G. Contreras, J.M. Gallardo, R. Garcia-Villegas, and J. Valdes. 1993. The making of a tight junction. J. Cell Sci. Suppl. 17:127-132.

3.Wegener, J., M. Sieber, and H.J. Galla. 1996. Impedance analysis of epithelial and endothelial cell monolayers cultured on gold surfaces. J. Biochem. Biophys. Methods 32:151-170.

4.Crone, C. and S.P. Olesen. 1982. Electrical resistance of brain microvascular endothelium. Brain Res. 241:49-55.

5.Claude, P. 1978. Morphological factors influencing transepithelial permeability: a model for the resistance of the zonula occludens. J. Membr. Biol. 39:219-232.

6.Powell, D.W. 1981. Barrier function of epithelia. Am. J. Physiol. 241:G275-G288.

7.Jovov, B., N.K. Wills, and S.A. Lewis. 1991. A spectroscopic method for assessing confluence of epithelial cell cultures. Am. J. Physiol. 261: C1196-C1203.

8.Lo, C.M., C.R. Keese, and I. Giaever. 1999. Cell-substrate contact: another factor may influence transepithelial electrical resistance of cell layers cultured on permeable filters. Exp. Cell Res. 250:576-580.

9.Franke, H., H. Galla, and C.T. Beuckmann. 2000. Primary cultures of brain microvessel endothelial cells: a valid and flexible model to study drug transport through the blood-brain barrier in vitro. Brain Res. Brain Res. Protoc. 5:248-256.

10.Hakvoort, A., M. Haselbach, J. Wegener, D. Hoheisel, and H.J. Galla. 1998. The polarity of choroid plexus epithelial cells in vitro is improved in serum-free medium. J. Neurochem. 71:1141-1150.

11.McAdams, E., A. Lackermeier, J. McLaughlin, D. Macken, and J. Jossinet. 1995. The linear and non-linear electrical properties of the electrode/electrolyte interface. Biosens. Bioelectron. 10:67-74

12.Pajkossy, T. 1994. Impedance of capacitive electrodes. J. Electroanal. Chem. 364:111.

13.Bevington, P.R. 1969. Data Reduction and Error Analysis for the Physical Science. McGraw-Hill, New York.

14.Wegener, J., A. Hakvoort, and H.J. Galla. 2000. Barrier function of porcine choroid plexus epithelial cells is modulated by cAMP-dependent pathways in vitro. Brain Res. 853:115-124.

15.Kottra, G., W. Haase, and E. Fromter. 1993. Tight-junction tightness of Necturus gall bladder epithelium is not regulated by cAMP or intracellular $\mathrm{Ca} 2+$. I. Microscopic and general electrophysiological observations. Pflugers Arch. 425:528-534.

16.Gath, U., A. Hakvoort, J. Wegener, S. Decker, and H.J. Galla. 1997. Porcine choroid plexus cells in culture: expression of polarized phenotype, maintenance of barrier properties and apical secretion of CSF-components. Eur. J. Cell Biol. 74:68-78.

17.Hoheisel, D., T. Nitz, H. Franke, J. Wegener, A. Hakvoort, T. Tilling, and H.J. Galla. 1998. Hydrocortisone reinforces the blood-brain barrier properties in a serum free cell culture system. Biochem. Biophys. Res. Commun. 244:312316.

Vol. 37, No. 4 (2004)
18.Lim, J.J. and J. Fischbarg. 1981. Electrical properties of rabbit corneal endothelium as determined from impedance measurements. Biophys. J. 36:677-695.

19.Wegener, J., S. Zink, P. Rosen, and H. Galla. 1999. Use of electrochemical impedance measurements to monitor beta- adrenergic stimulation of bovine aortic endothelial cells. Pflugers Arch. 437:925-934.
Received 6 March 2004; accepted 29 June 2004.

Address correspondence to Joachim Wegener, Institut für Biochemie, Westf. Wilhelms-Universität Münster, Wilhelm-Klemm Str. 2, 48149 Münster, Germany. e-mail: wegenej@uni-muenster.de 